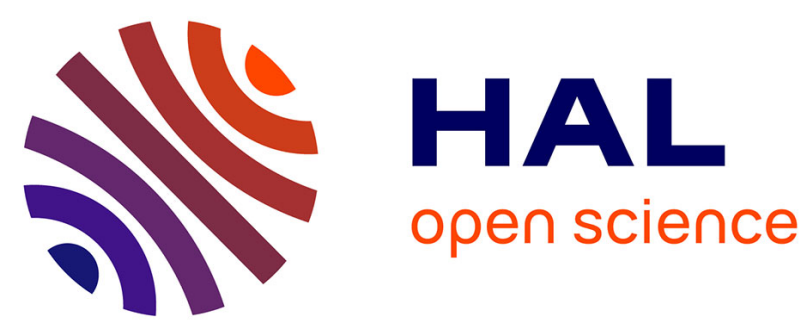

\title{
Comparaison biomécanique du comportement en fatigue de greffes quatre brins faufilées et non faufilées pour la reconstruction du ligament croisé antérieur
}

\author{
Laure-Lise Gras, Antoine Combes
}

\section{To cite this version:}

Laure-Lise Gras, Antoine Combes. Comparaison biomécanique du comportement en fatigue de greffes quatre brins faufilées et non faufilées pour la reconstruction du ligament croisé antérieur. SFA 2016 Congrès annuel de la Société Francophone d'Arthroscopie, Nov 2016, PARIS, France. pp.S275-S276, 10.1016/j.rcot.2016.10.013 . hal-01844570

\author{
HAL Id: hal-01844570 \\ https://hal.science/hal-01844570
}

Submitted on 19 Jul 2018

HAL is a multi-disciplinary open access archive for the deposit and dissemination of scientific research documents, whether they are published or not. The documents may come from teaching and research institutions in France or abroad, or from public or private research centers.
L'archive ouverte pluridisciplinaire HAL, est destinée au dépôt et à la diffusion de documents scientifiques de niveau recherche, publiés ou non, émanant des établissements d'enseignement et de recherche français ou étrangers, des laboratoires publics ou privés. 


\section{Communication particulière}

Comparaison biomécanique du comportement en fatigue de greffes quatre brins faufilées et non faufilées pour la reconstruction du ligament croisé antérieur

Biomechanical comparison of strength and fatigue resistance of continuous suture and no continuous suture grafts for anterior cruciate ligament reconstruction

Laure-Lise Gras ${ }^{* a, b, c}$, Antoine Combes ${ }^{d}$

* Premier auteur, auteur orateur

a. Université de Lyon, F-69622, Lyon, France ;

b. Université Claude Bernard Lyon 1, Villeurbanne ;

c. IFSTTAR, UMR_T9406, LBMC Laboratoire de Biomécanique et Mécanique des Chocs, F69675, Bron

;

d. Centre de chirurgie orthopédique Fleming, F38300, Bourgoin Jallieu

Mots clés: Reconstruction du ligament croisé antérieur, droit interne, demi-tendineux, expérimentation in vitro, propriétés mécaniques, faufilage, greffe quatre brins

Introduction : Dans le cadre de la reconstruction du ligament croisé antérieur au demi-tendineux quatre brins (DT4) se pose la question de la technique utilisée pour le créer. Nous proposons de comparer mécaniquement des greffes quatre brins obtenues avec les tendons des muscles ischiojambiers - droit interne (DI) et demi-tendineux (DT) - faufilées (F) et non faufilées (NF).

Matériel et méthodes : Les tendons DI et DT sont prélevés sur six sujets cadavériques embaumés. Des greffes à quatre brins sont réalisées : 12 DI et 12 DT. Les greffes du côté droit sont NF, celles du côté gauche sont $\mathrm{F}$. Chaque greffe est testée selon le même protocole expérimental. La greffe est placée sur une machine de traction. Une pré-charge de $1 \mathrm{~N}$ lui est appliquée puis l'effort est amené à $50 \mathrm{~N}$ et maintenu pendant 30s. Mille cycles de chargement-déchargement d'amplitude $200 \mathrm{~N}$ sont ensuite appliqués à la fréquence de $1 \mathrm{~Hz}$. Enfin, la greffe est allongée jusqu'à rupture à la vitesse de $20 \mathrm{~mm} / \mathrm{min}$. L'évolution de l'allongement de la greffe au cours des cycles, la raideur avant rupture et la charge à rupture sont étudiées et comparées avec une étude statistique.

Résultats : Au $1000^{\text {ème }}$ cycle, l'allongement des greffes NF (moyenne : $4.20 \mathrm{~mm}$; DTNF : $3.51 \mathrm{~mm}$ ) est supérieur aux greffes $F$ (moyenne: $3.44 \mathrm{~mm}$; DTF : $3.17 \mathrm{~mm}$ ), mais ces différences ne sont pas statistiquement significatives. La raideur avant rupture pour les greffes NF (moyenne : $307 \mathrm{~N} / \mathrm{mm}$; DTNF : $336 \mathrm{~N} / \mathrm{mm}$ ) est inférieure à celle des greffes $\mathrm{F}$ (moyenne : $322 \mathrm{~N} / \mathrm{mm}$; DTF : $343 \mathrm{~N} / \mathrm{mm}$ ), mais cette différence $n$ 'est pas significative. La charge à rupture est statistiquement différente entre les greffes NF (moyenne : 843N ; DTNF : 924N) et F (moyenne : 1133N ; DTF : 1263N) ( $p$-value < 0.01 ).

Discussion: Les tendons étant prélevés sur sujets embaumés, leurs propriétés mécaniques sont altérées, néanmoins, cette limitation n'empêche pas la comparaison NF/F. L'absence de différence significative peut-être expliquée par le nombre limité de sujets malgré l'utilisation des deux ischiojambiers.

Conclusion : Au regard des résultats, la greffe faufilée semble préférable. 